\title{
Robust Maximization of Consumption with Logarithmic Utility
}

\section{Daniel Hernández-Hernández*} Alexander Schied**

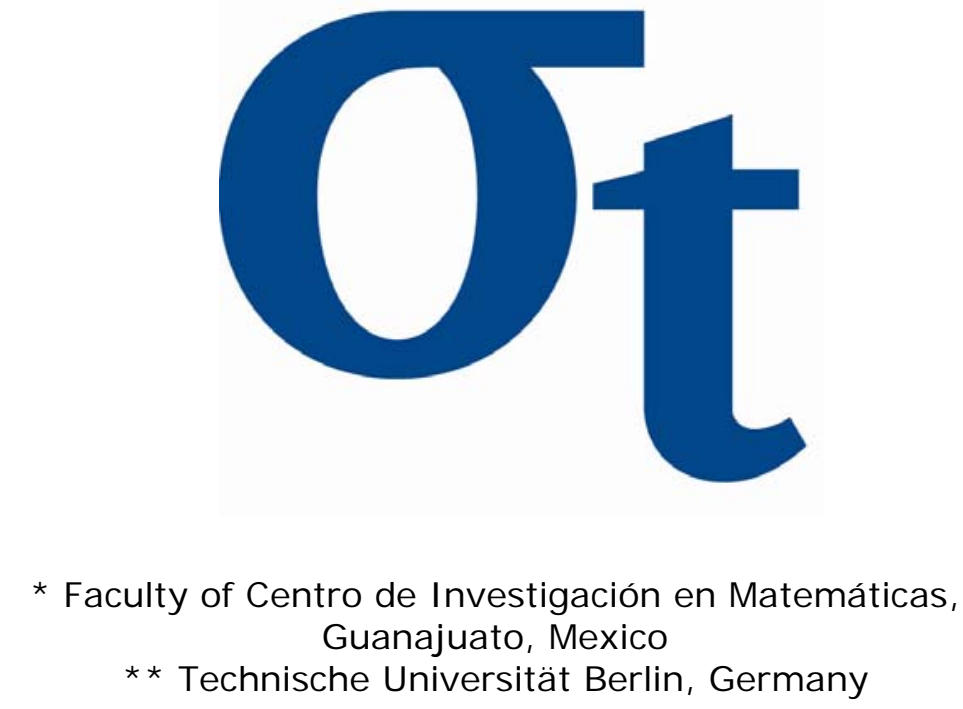

This research was supported by the Deutsche Forschungsgemeinschaft through the SFB 649 "Economic Risk". 


\title{
Robust maximization of consumption with logarithmic utility
}

\author{
Daniel Hernández-Hernández and Alexander Schied*
}

\begin{abstract}
We analyze the stochastic control approach to the dynamic maximization of the robust utility of consumption and investment. The robust utility functionals are defined in terms of logarithmic utility and a dynamically consistent convex risk measure. The underlying market is modeled by a diffusion process whose coefficients are driven by an external stochastic factor process. Our main results give conditions on the minimal penalty function of the robust utility functional under which the value function of our problem can be identified with the unique classical solution of a quasilinear PDE within a class of functions satisfying certain growth conditions.
\end{abstract}

\section{INTRODUCTION}

One of the fundamental problems in mathematical finance and mathematical economics is the construction of investment strategies that maximize the utility functional of a risk-averse investor. In the majority of the corresponding literature, the optimality criterion is based on a classical expected utility functional of von Neumann-Morgenstern form, which requires the choice of a single probabilistic model $\mathbb{P}$. In reality, however, the choice of $\mathbb{P}$ is often subject to model uncertainty. Schmeidler [?] and Gilboa and Schmeidler [10] therefore proposed the use of robust utility functionals of the form

$$
X \longmapsto \inf _{Q \in \mathcal{Q}} E_{Q}[U(X)],
$$

where $\mathcal{Q}$ is a set of prior probability measures. In analogy to the move from coherent to convex risk measures, Maccheroni et al. [16] recently suggested to model investor's preferences by robust utility functionals of the form

$$
X \longmapsto \inf _{Q}\left(E_{Q}[U(X)]+\gamma(Q)\right),
$$

where $\gamma$ is a penalty function defined on the set of all possible probabilistic models.

Optimal investment problems for robust utility functionals (1) were considered, among others, by Talay and Zheng [24], Quenez [18], Schied [19], Burgert and Rüschendorf [3], Schied and $\mathrm{Wu}$ [23], Föllmer and Gundel [8], and the authors [13]. For the generalized utility functionals of type (2), the most popular choice for the penalty function has so far been the entropic penalty function $\gamma(Q)=k H(Q \mid \mathbb{P})$ for a constant $k>0$ and a reference probability measure $\mathbb{P}$; see, e.g., Hansen and Sargent [12] and Bordigoni et al.

\footnotetext{
*Supported by Deutsche Forschungsgemeinschaft through the SFB 649 "Economic Risk".

Daniel Hernández-Hernández is with Faculty of Centro de Investigación en Matemáticas, Apartado Postal 402, Guanajuato, Gto. C.P. 36000, México dherecimat.mx

Alexander Schied is with Faculty of Institute of Mathematics, MA 7-4, Berlin University of Technology, Strasse des 17. Juni 136, 10623 Berlin, Germany schiedemath.tu-berlin. de
}

[2]. The duality theory for the optimal investment problem with a general penalty function $\gamma$ was developed by Schied [21]. It was extended by Wittmüss [25] to the consumptioninvestment problem with random endowment.

In this paper, we propose a stochastic control approach to the dynamic maximization of robust utility functionals of the form (2). The penalty function $\gamma$ will be defined in a Brownian setting and, apart from certain basic requirements such as time consistency, has a rather general form. In particular, we will go beyond the very particular situation of entropic penalties and include the 'coherent' setting (1) as a special case. Our setting will involve logarithmic utility $U(x)=\log x$ and an incomplete financial market model, whose volatility, interest rate process, and trend are driven by an external stochastic factor process. In this setting, the control approach to the optimization of the terminal wealth was developed by the authors in a previous paper [14]. We now extend these results by also allowing for intertemporal consumption.

Our goal consists in characterizing the value function and the optimal investment strategy via the solution of a quasilinear Hamilton-Jacobi-Bellman PDE. As a byproduct, we also obtain a formula for the least-favorable martingale measure in the sense of Föllmer and Gundel [8]. In contrast to earlier approaches such as [24], we avoid the use of viscosity solutions and concentrate our effort on obtaining strong regularity results, which allow us to identify the value function as a unique classical solution of the PDE in question. Regularity of solutions is important because it facilitates the use of standard numerical methods for solving the PDE, and we will use such methods in illustrating some interesting qualitative properties of the optimal strategy.

Our method consists in combining the duality results from [21] and [25] with a PDE approach to the dual problem of determining optimal martingale measures. This technique has already been applied successfully by Castañeda-Leyva and Hernández-Hernández [4], [5] to the maximization of von Neumann-Morgenstern expected utility and by the authors [13] and Schied [22] in the maximization of 'coherent' robust utility functionals of the form (1). The approach has also been applied successfully to the maximization of the robust utility (2) of the terminal wealth; see [14]. In this paper, we extend the results from [14] to the case of combined intertemporal consumption and investment.

In the next section we describe the set-up of our problem and state the theorems for our main findings. Their proofs can be obtained by appropriately adapting the proofs in [13]. Specifically, one has to replace the duality results from [21] by those from [25]. In setting up the control approach to the 
dual problem and in identifying the optimal consumptioninvestment strategy, one can follow the arguments in [22].

\section{STATEMENT OF MAIN RESULTS}

We consider a financial market model with a locally riskless money market account

$$
d S_{t}^{0}=S_{t}^{0} r\left(Y_{t}\right) d t
$$

and a risky asset defined under a reference measure $\mathbb{P}$ through the SDE

$$
d S_{t}=S_{t} b\left(Y_{t}\right) d t+S_{t} \sigma\left(Y_{t}\right) d W_{t}^{1} .
$$

Here $W^{1}$ is a standard $\mathbb{P}$-Brownian motion and $Y$ denotes an external economic factor process modeled by the SDE

$$
d Y_{t}=g\left(Y_{t}\right) d t+\rho d W_{t}^{1}+\bar{\rho} d W_{t}^{2},
$$

where $\rho \in[-1,1]$ is some correlation factor, $\bar{\rho}:=\sqrt{1-\rho^{2}}$, and $W^{2}$ is a standard $\mathbb{P}$-Brownian motion, which is independent of $W^{1}$ under $\mathbb{P}$. We suppose that the economic factor cannot be traded directly so that the market model will typically be incomplete.

We assume that $g(\cdot)$ is in $C^{2}(\mathbb{R})$ with derivative $g^{\prime} \in$ $C_{b}^{1}(\mathbb{R})$, and $r(\cdot), b(\cdot)$, and $\sigma(\cdot)$ belong to $C_{b}^{2}(\mathbb{R})$, where $C_{b}^{k}(\mathbb{R})$ denotes the class of bounded functions with bounded derivatives up to order $k$. The 'market price of risk' is defined via the function

$$
\theta(y):=\frac{b(y)-r(y)}{\sigma(y)},
$$

and we will assume that $\sigma(\cdot) \geq \sigma_{0}>0$ for some constant $\sigma_{0}$. The assumption of time-independent coefficients is for notational convenience only and can easily be relaxed.

In most economic situations, investors typically face model uncertainty in the sense that the dynamics of the relevant quantities are not precisely known. One common approach to coping with model uncertainty is to allow in principle all probability models corresponding to probability measures $Q \ll \mathbb{P}$ and to penalize each such model with a penalty $\gamma(Q)$. To define $\gamma(Q)$, we assume henceforth that everything is modeled on the canonical path space $\left(\Omega, \mathcal{F},\left(\mathcal{F}_{t}\right)\right)$ of $W=$ $\left(W^{1}, W^{2}\right)$. Then every probability measure $Q \ll \mathbb{P}$ admits a progressively measurable process $\eta=\left(\eta_{1}, \eta_{2}\right)$ such that

$$
\frac{d Q}{d \mathbb{P}}=\mathcal{E}\left(\int_{0} \eta_{1 t} d W_{t}^{1}+\int_{0} \eta_{2 t} d W_{t}^{2}\right)_{T} \quad Q-\text { a.s. },
$$

where $\mathcal{E}(M)_{t}=\exp \left(M_{t}-\langle M\rangle_{t} / 2\right)$ denotes the DoleansDade exponential of a continuous semimartingale $M$. Such a measure $Q$ will receive a penalty

$$
\gamma(Q):=E_{Q}\left[\int_{0}^{T} h\left(\eta_{t}\right) d t\right]
$$

where $h: \mathbb{R}^{2} \rightarrow[0, \infty]$ is convex and lower semicontinuous. For simplicity, we will suppose $h(0)=0$ so that $\gamma(\mathbb{P})=0$. We will also assume that $h$ is continuously differentiable on its effective domain dom $h:=\left\{\eta \in \mathbb{R}^{2} \mid h(\eta)<\infty\right\}$ and satisfies the coercivity condition

$$
h(x) \geq \kappa_{1}|x|^{2}-\kappa_{2} \quad \text { for constants } \kappa_{1}, \kappa_{2}>0 .
$$

The choice $h(x)=|x|^{2} / 2$ corresponds to the entropic penalty function considered in Hansen and Sargent [12] and Bordigoni et al. [2]; see Remark 2.6 below. Again, our assumption that $h$ does not depend on time is for notational convenience only.

Let $\mathcal{A}$ denote the set of all pairs $(c, \pi)$ of progressively measurable processes such that $c \geq 0, \int_{0}^{T} c_{s} d s<\infty$, and $\int_{0}^{T} \pi_{s}^{2} d s<\infty \mathbb{P}$-a.s. For $(c, \pi) \in \mathcal{A}$ and $x>0$, we define $X^{x, c, \pi}$ as the unique solution of the linear SDE

$d X_{t}^{x, c, \pi}=\frac{X_{s}^{x, c, \pi}\left(1-\pi_{s}\right)}{S_{s}^{0}} d S_{s}^{0}+\frac{X_{s}^{x, c, \pi} \pi_{s}}{S_{s}} d S_{s}-c_{s} d s$

with initial value $X_{0}^{x, c, \pi}=x$. The process $X^{x, c, \pi}$ thus describes the evolution of the wealth process of an investor with initial endowment $X_{0}^{x, c, \pi}=x>0$ investing the fraction $\pi_{s}$ of the current wealth into the risky asset at time $s \in[0, T]$. By $\mathcal{A}(x)$ we denote the subclass of all $(c, \pi) \in \mathcal{A}$ that are admissible in the sense that $X_{t}^{x, c, \pi} \geq 0 \mathbb{P}$-a.s. for all $t$.

The objective of the investor is to maximize

$$
\inf _{Q \ll \mathbb{P}}\left(E_{Q}\left[\int_{0}^{T} U\left(c_{t}\right) d t+U\left(X_{T}^{x, c, \pi}\right)\right]+\gamma(Q)\right)
$$

over $(c, \pi) \in \mathcal{A}(x)$. The utility function $U:(0, \infty) \rightarrow \mathbb{R}$ will be specified in the sequel as a HARA utility function with risk aversion parameter $\alpha=0$, i.e.,

$$
U(x)=\log x .
$$

Our goal is to characterize the value function

$$
\begin{aligned}
& u(x):= \\
& \quad \sup _{\pi \in \mathcal{A}} \inf _{Q \ll \mathbb{P}}\left(E_{Q}\left[\int_{0}^{T} \log c_{t} d t+\log X_{T}^{x, c, \pi}\right]+\gamma(Q)\right)
\end{aligned}
$$

of the robust utility maximization problem (6) in terms of the solution $v$ of the quasi-linear parabolic initial value problem

$$
\left\{\begin{array}{l}
v_{t}=\frac{1}{2} v_{y y}+\phi\left(v_{y}\right)+g v_{y}+(1+t) r \\
v(0, \cdot)=0
\end{array}\right.
$$

where the nonlinearity $\phi\left(v_{y}\right)=\phi\left(t, y, v_{y}(t, y)\right)$ is given by

$$
\phi(t, y, z):=\psi(t, y,(\rho, \bar{\rho}) z) \quad y, z \in \mathbb{R} .
$$

for the function

$$
\psi(t, y, x):=\inf _{\eta \in \mathbb{R}^{2}}\left\{\eta \cdot x+\frac{1}{2}(1+t)\left(\eta_{1}+\theta(y)\right)^{2}+h(\eta)\right\},
$$

with $y \in \mathbb{R}, x \in \mathbb{R}^{2}$. Here, $\eta \cdot x$ denotes the inner product of $\eta$ and $x$. The easy case is the one in which the effective domain of $h$ is compact:

Theorem 2.1: Suppose that dom $h$ is compact. Then the value function $u$ of the robust utility maximization problem satisfies

$$
u(x)=(1+T) \log x+v\left(T, Y_{0}\right),
$$

where $v:[0, T] \times \mathbb{R} \rightarrow \mathbb{R}$ is the unique classical solution to (8) within the class of functions in $C^{1,2}((0, T) \times \mathbb{R}) \cap$ $C([0, T] \times \mathbb{R})$ satisfying a polynomial growth condition.

Suppose furthermore that $\eta^{*}:[0, T] \times \mathbb{R} \rightarrow \mathbb{R}$ is a measurable function such that $\eta^{*}(t, y)$ belongs to the 
supergradient of the concave function $x \mapsto \psi(t, y, x)$ at $x=(\rho, \bar{\rho}) v_{y}(t, y)$. Then an optimal strategy $(\widehat{c}, \widehat{\pi}) \in \mathcal{A}(x)$ for the robust problem can be obtained by letting

$$
\widehat{\pi}_{t}=\frac{\eta_{1}^{*}\left(T-t, Y_{t}\right)+\theta\left(Y_{t}\right)}{\sigma\left(Y_{t}\right)}
$$

and by consuming at a rate proportional to the current total wealth $X_{t}^{x, \widehat{c}, \widehat{\pi}}$ :

$$
\widehat{c}_{t}=\frac{1}{1+T-t} X_{t}^{x, \widehat{c}, \widehat{\pi}}
$$

Moreover, by defining a measure $\widehat{Q} \sim \mathbb{P}$ via

$$
\frac{d \widehat{Q}}{d \mathbb{P}}=\mathcal{E}\left(\int_{0} \eta^{*}\left(T-t, Y_{t}\right) d W_{t}\right)_{T}
$$

we obtain a saddlepoint $(\widehat{\pi}, \widehat{Q})$ for the maximin problem (6).

The regularity of the value function obtained in the preceding theorem is important, because it facilitates the use of standard numerical methods for solving the PDE (8).

Remark 2.2: The proof of Theorem 2.1 shows that the probability measure $P^{*}$ with density

$$
\frac{d P^{*}}{d \mathbb{P}}=\mathcal{E}\left(-\int_{0} \theta\left(Y_{s}\right) d W_{s}^{1}+\int_{0} \eta_{2}^{*}\left(T-s, Y_{s}\right) d W_{s}^{2}\right)_{T}
$$

is a least favorable martingale measure in the sense of Föllmer and Gundel [8]. This will also be true in the setting of Theorems 2.3 and 2.5 .

The problem becomes more difficult when $\operatorname{dom} h$ is noncompact, because then we can no longer apply standard theorems on the existence of classical solutions to (8). Other difficulties appear when dom $h$ is not only noncompact but also unbounded. For instance, we may have $\gamma(Q)<\infty$ even if $Q$ is not equivalent but merely absolutely continuous with respect to $\mathbb{P}$, and this leads to difficulties when one tries to work directly on the primal problem. Moreover, since the optimal $\eta^{*}$ takes values in the unbounded set dom $h$, one needs an additional argument to ensure that the stochastic exponential in (9) is a true martingale and hence defines a probability measure $\widehat{Q} \ll \mathbb{P}$. Our strategy to get the necessary integrability of the process $\eta_{1}^{*}\left(T-t, Y_{t}\right)$ is to use qualitative properties of solutions $v$ to (8) as to control the growth of the gradient $v_{y}$. In doing so, we have to eliminate the possible competition between the linear term $g v_{y}$ and the nonlinear term $\phi\left(v_{y}\right)$ by imposing a growth condition on $\phi$.

Theorem 2.3: Suppose that $g$ is bounded and that there exists some $\varepsilon>0$ such that

$$
\liminf _{|p| \rightarrow \infty}\left|\frac{\phi(t, y, p)}{p}\right| \geq \varepsilon+|g(y)| .
$$

Then the value function $u$ of the robust utility maximization problem satisfies $u(x)=(1+T) \log x+v\left(T, Y_{0}\right)$ where $v$ is the unique classical solution of (8) within the class of functions in $C^{1,2}((0, T) \times \mathbb{R}) \cap C([0, T] \times \mathbb{R})$ with bounded gradient $v_{y}$. Under these conditions, also the conclusions on the optimal strategy $(\widehat{c}, \widehat{\pi})$ and the measure $\widehat{Q}$ in Theorem 2.1 remain true.
The most interesting case is the one in which both dom $h$ and the function $g$ are unbounded. Here we need an additional condition on the shape of the function $\psi$. Note that $g$ is unbounded if, e.g., $Y$ is an Ornstein-Uhlenbeck process.

Definition 2.4: Let $f: \mathbb{R}^{2} \rightarrow \mathbb{R}$ be an upper semicontinuous concave function. We will say that $f$ satisfies a radial growth condition in direction $x \in \mathbb{R}^{2}$ if there exist positive constants $p_{0}$ and $C$ such that

$\max \{|z| \mid z \in \partial f(p x)\} \leq C\left(1+\left|\partial_{p}^{+} f(p x)\right| \vee\left|\partial_{p}^{-} f(p x)\right|\right)$,

for $p \in \mathbb{R},|p| \geq p_{0}$, where $\partial f(p x)$ denotes the supergradient of $f$ in $p x$ and $\partial_{p}^{+} f(p x)$ and $\partial_{p}^{-} f(p x)$ are the right-hand and left-hand derivatives of the concave function $p \mapsto f(p x)$.

Note that if $f$ is of the form $f(x)=f_{0}(|x|)$ for some convex increasing function $f_{0}$, then the radial growth condition is satisfied in any direction $x \neq 0$ with constant $C=1$.

Theorem 2.5: Suppose that $|\phi(t, y, p) / p| \rightarrow \infty$ as $|p| \rightarrow$ $\infty$ and assume that $\psi(t, y, \cdot)$ satisfies a radial growth condition in direction $(\rho, \bar{\rho})$, uniformly in $y$ and $t$. Then the value function $u$ of the robust utility maximization problem satisfies $u(x)=(1+T) \log x+v\left(T, Y_{0}\right)$ where $v$ is the unique classical solution of (8) within the class of polynomially growing functions in $C^{1,2}((0, T) \times \mathbb{R}) \cap C([0, T] \times \mathbb{R})$ whose gradient satisfies a growth condition of the form

$$
\left|\partial_{p}^{-} \phi\left(y ; v_{y}(t, y)\right)\right| \vee\left|\partial_{p}^{+} \phi\left(y ; v_{y}(t, y)\right)\right| \leq C_{1}(1+|y|)
$$

for some constant $C_{1}$. Under these conditions, also the conclusions on the optimal strategy $(\widehat{c}, \widehat{\pi})$ and the measure $\widehat{Q}$ in Theorem 2.1 remain true.

Remark 2.6: For $q>0$, the choice $h(x)=\frac{1}{2 q}|x|^{2}$ corresponds to the penalty function $\gamma(Q)=\frac{1}{q} H(Q \mid \mathbb{P})$, where

$H(Q \mid \mathbb{P})=\int \frac{d Q}{d \mathbb{P}} \log \frac{d Q}{d \mathbb{P}} d \mathbb{P}=\sup _{Y \in L^{\infty}}\left(E_{Q}[Y]-\log \mathbb{E}\left[e^{Y}\right]\right)$

is the relative entropy of $Q$ with respect to $\mathbb{P}$. Due to the classical duality formula

$$
\log \mathbb{E}\left[e^{X}\right]=\sup _{Q \in \mathcal{Q}}\left(E_{Q}[X]-H(Q \mid \mathbb{P})\right)
$$

the above choices correspond to the utility functional

$$
\inf _{Q \ll \mathbb{P}}\left(E_{Q}[\log X]+\gamma(Q)\right)=-\frac{1}{q} \log \mathbb{E}\left[e^{-q \log X}\right] .
$$

Hence, as long as there is no consumption, the robust utility maximization problem (6) is equivalent to the maximization of the standard expected utility $\mathbb{E}\left[U\left(X_{T}^{x, c, \pi}\right)\right]$ for the HARA utility function $U(x)=-x^{-q}$. With nontrivial consumption, however, such a reduction is no longer possible, and our problem can no longer be formulated exclusively in terms of standard expected utility. This situation problem is covered as a special case of Theorem 2.5. Indeed, the function $\psi$ has the quadratic form

$$
\psi(t, y, x)=-\frac{1}{2}\left(\frac{(1+t) q}{1+q}\left(x_{1}+\theta(y)\right)^{2}+q x_{2}^{2}-\theta(y)^{2}\right),
$$

and it is easily checked that it satisfies the radial growth condition in any direction. See Hansen and Sargent [12] and 
Bordigoni et al. [2] for earlier studies of the problem of optimal consumption with entropic penalties.

\section{ACKNOWLEDGMENTS}

Acknowledgement: Part of this work was done while the first author visited INRIA Rocquencourt, its support trough the project Mathfi is greatly appreciated as well as its hospitality. The second author is grateful for support and hospitality during a research visit at the National Center for Theoretical Sciences at Tsing-Hua University and the Academia Sinica in Taipei, where part of the research for this paper was conducted. He is indebted to Shuenn-Jyi Sheu and Ching-Tang Wu for many stimulating discussions.

\section{REFERENCES}

[1] Barrieu, P., El Karoui, N. Optimal Derivatives Design under Dynamic Risk Measures. In: Mathematics of finance, 13-25, Contemp. Math., 351, Amer. Math. Soc., Providence, RI, 2004.

[2] Bordigoni, G. Matoussi, A. Schweizer, M. A stochastic control approach to a robust utility maximization problem. To appear in Proceedings of Abel Symposium 2005, Springer.

[3] Burgert, C., Rüschendorf, L. Optimal consumption strategies under model uncertainty. Statist. Decisions 23 (2005), no. 1, 1-14.

[4] Castañeda-Leyva, N., Hernández-Hernández D. Optimal investment in incomplete financial markets with stochastic volatility. Contemp. Math. 336, 119-136 (2003).

[5] Castañeda-Leyva, N., Hernández-Hernández D. Optimal consumptioninvestment problems in incomplete markets with stochastic coefficients. SIAM J. Control Optim. 44, No. 4, pp. 1322-1344 (2005).

[6] Fleming, W., Hernández-Hernández, D. Tradeoff between consumption and investments in incomplete financial markets. Appl. Math. Optim. 52 (2005), 219-235.

[7] Fleming, W., Soner, M. Controlled Markov processes and viscosity solutions. Springer-Verlag, New York, 1993. Second edition 2006.

[8] Föllmer, H., Gundel, A. Robust projections in the class of martingale measures. Illinois J. Math. 50, 439-472 (2006).

[9] Föllmer, H., Schied, A. Stochastic Finance: An Introduction in Discrete Time. Berlin: de Gruyter Studies in Mathematics 27 (2002). Second edition (2004).

[10] Gilboa, I., Schmeidler, D. Maximin expected utility with non-unique prior. J. Math. Econ. 18, 141-153 (1989).

[11] Gundel, A. Weber S. Robust Utility Maximization with Limited Downside Risk in Incomplete Markets. Preprint, Cornell University (2006).

[12] Hansen, L., Sargent, T. Robust control and model uncertainty. American Economic Review 91, 60-66 (2001).

[13] Hernández-Hernández D., Schied, A. Robust utility maximization in a stochastic factor model. Stat. Decisions 24, 109-125 (2006).

[14] Hernández-Hernández D., Schied, A. A control approach to robust utility maximization with logarithmic utility and time consistent penalties. Forthcoming in Stoch. Proc. Appl.

[15] Klöppel, S., Schweizer, M. Dynamic indifference valuation via convex risk measures. To appear in Math. Finance (2006).

[16] Maccheroni, F., Marinacci, M., Rustichini, A. Ambiguity aversion, malevolent nature, and the variational representation of preferences. To appear in Econometrica.

[17] Kushner, H., Dupuis, P. Numerical methods for stochastic control problems in continuous time. Second edition. Applications of Mathematics (New York), 24. Stochastic Modelling and Applied Probability. Springer-Verlag, New York, 2001.

[18] Quenez, M.-C. Optimal portfolio in a multiple-priors model. Seminar on Stochastic Analysis, Random Fields and Applications IV, 291-321, Progr. Probab., 58, Birkhäuser, Basel, 2004.

[19] Schied, A. Optimal investments for robust utility functionals in complete market models. Math. Oper. Research. 30, No. 3, 750-764 (2005).

[20] Schied, A. Risk measures and robust optimization problems. Stochastic Models, 22, 753-831 (2006).

[21] Schied, A. Optimal investments for risk- and ambiguity-averse preferences: a duality approach. Finance Stochastics 11, No. 1, 107-129 (2007).
[22] Schied, A. Robust control of consumption-investment strategies in a stochastic factor model. Preprint, TU Berlin (2007).

[23] Schied, A., Wu, C.-T. Duality theory for optimal investments under model uncertainty. Stat. Decisions 23, No. 3, 199-217 (2005).

[24] Talay, D., Zheng, Z. Worst case model risk management. Finance Stochast. 6, 517-537 (2002).

[25] Wittmüss, W. Robust optimization of consumption with random endowment. Preprint, TU Berlin (2007). 


\section{SFB 649 Discussion Paper Series 2007}

For a complete list of Discussion Papers published by the SFB 649, please visit http://sfb649.wiwi.hu-berlin.de.

001 "Trade Liberalisation, Process and Product Innovation, and Relative Skill Demand" by Sebastian Braun, January 2007.

002 "Robust Risk Management. Accounting for Nonstationarity and Heavy Tails" by Ying Chen and Vladimir Spokoiny, January 2007.

003 "Explaining Asset Prices with External Habits and Wage Rigidities in a DSGE Model." by Harald Uhlig, January 2007.

004 "Volatility and Causality in Asia Pacific Financial Markets" by Enzo Weber, January 2007.

005 "Quantile Sieve Estimates For Time Series" by Jürgen Franke, JeanPierre Stockis and Joseph Tadjuidje, February 2007.

006 "Real Origins of the Great Depression: Monopolistic Competition, Union Power, and the American Business Cycle in the 1920s" by Monique Ebell and Albrecht Ritschl, February 2007.

007 "Rules, Discretion or Reputation? Monetary Policies and the Efficiency of Financial Markets in Germany, 14th to 16th Centuries" by Oliver Volckart, February 2007.

008 "Sectoral Transformation, Turbulence, and Labour Market Dynamics in Germany" by Ronald Bachmann and Michael C. Burda, February 2007.

009 "Union Wage Compression in a Right-to-Manage Model" by Thorsten Vogel, February 2007.

010 "On $\sigma$-additive robust representation of convex risk measures for unbounded financial positions in the presence of uncertainty about the market model" by Volker Krätschmer, March 2007.

011 "Media Coverage and Macroeconomic Information Processing" by Alexandra Niessen, March 2007.

012 "Are Correlations Constant Over Time? Application of the CC-TRIG to Return Series from Different Asset Classes." by Matthias Fischer, March 2007.

013 "Uncertain Paternity, Mating Market Failure, and the Institution of Marriage" by Dirk Bethmann and Michael Kvasnicka, March 2007.

014 "What Happened to the Transatlantic Capital Market Relations?" by Enzo Weber, March 2007.

015 "Who Leads Financial Markets?" by Enzo Weber, April 2007.

016 "Fiscal Policy Rules in Practice" by Andreas Thams, April 2007.

017 "Empirical Pricing Kernels and Investor Preferences" by Kai Detlefsen, Wolfgang Härdle and Rouslan Moro, April 2007.

018 "Simultaneous Causality in International Trade" by Enzo Weber, April 2007.

019 "Regional and Outward Economic Integration in South-East Asia" by Enzo Weber, April 2007.

020 "Computational Statistics and Data Visualization" by Antony Unwin, Chun-houh Chen and Wolfgang Härdle, April 2007.

021 "Ideology Without Ideologists" by Lydia Mechtenberg, April 2007.

022 "A Generalized ARFIMA Process with Markov-Switching Fractional Differencing Parameter" by Wen-Jen Tsay and Wolfgang Härdle, April 2007.

\section{SFB 649, Spandauer Straße 1, D-10178 Berlin} http:/ / sfb649.wiwi.hu-berlin.de 
023 "Time Series Modelling with Semiparametric Factor Dynamics" by Szymon Borak, Wolfgang Härdle, Enno Mammen and Byeong U. Park, April 2007.

024 "From Animal Baits to Investors' Preference: Estimating and Demixing of the Weight Function in Semiparametric Models for Biased Samples" by Ya'acov Ritov and Wolfgang Härdle, May 2007.

025 "Statistics of Risk Aversion" by Enzo Giacomini and Wolfgang Härdle, May 2007.

026 "Robust Optimal Control for a Consumption-Investment Problem" by Alexander Schied, May 2007.

027 "Long Memory Persistence in the Factor of Implied Volatility Dynamics" by Wolfgang Härdle and Julius Mungo, May 2007.

028 "Macroeconomic Policy in a Heterogeneous Monetary Union" by Oliver Grimm and Stefan Ried, May 2007.

029 "Comparison of Panel Cointegration Tests" by Deniz Dilan Karaman Örsal, May 2007.

030 "Robust Maximization of Consumption with Logarithmic Utility" by Daniel Hernández-Hernández and Alexander Schied, May 2007. 